\title{
Metabolic Specialization in Fast and Slow Muscle Fibers of the Developing Rat
}

\author{
Patti M. Nemeth, ${ }^{1}$ Beverly J. Norris, ${ }^{1}$ Lata Solanki, ${ }^{1}$ and Alan M. Kelly ${ }^{2}$ \\ Departments of 'Neurology and of Anatomy and Neurobiology, School of Medicine, Washington University, St. Louis, \\ Missouri 63110, and 'Laboratory of Pathology, School of Veterinary Medicine, University of Pennsylvania, Philadelphia, \\ Pennsylvania 19104
}

Individual fibers of prospective fast (extensor digitorum longus; EDL) and slow (soleus) muscles of rats have been analyzed to determine the profiles of key energy-generating enzymes at successive stages of postnatal development. Mean activities of lactate dehydrogenase (LDH) and adenylokinase (AK), 2 enzymes associated with contractile function, are significantly different in the 2 fiber populations at birth; furthermore, wide variations in enzyme activities exist among the individual fibers. There is a progressive refinement of enzyme levels in the soleus into a more uniform fiber population, while the fibers in the EDL progressively diverge into 2 distinct phenotypes. Changes in EDL and soleus are punctuated by periods of rapid change, with the period between 10 and $21 \mathrm{~d}$ being most eventful. Generally, the maturation profiles of LDH and AK coincide with the transition from neonatal to adult fast myosins and closely reflect the timing of energy demands imposed by contractile activity patterns.

In contrast, activities of the oxidative enzymes malate dehydrogenase and $\beta$-hydroxyacyl CoA dehydrogenase are similar in both muscles at birth and steadily increase during the first 3 weeks, suggesting a progressive adaptation to the aerobic extrauterine environment. After $\mathbf{3 0} \mathrm{d}$, there are differential changes in the oxidative profiles of enzymes for fatty acid and glucose metabolism. The profiles follow dietary changes associated with weaning, which suggests a phenotypic dependence of neonatal muscle on the particular available energy substrate.

All enzymes are low in all fibers of EDL and soleus at birth, indicating their modest metabolic capacity. Experimental alterations In thyrold function show that hyperthyroldlsm causes an accelerated maturation of all enzyme levels and that, generally, hypothyroidism causes an inhibition or restriction of maturation.

\footnotetext{
Received Aug. 17, 1988; revised Dec. 29, 1988; accepted Jan. 13, 1989.

This research was supported by National Institutes of Health grants DK 38375 and HL 15835 and grants from the Muscular Dystrophy Association of America. We wish to express our appreciation to Mrs. Patti Nacci for preparing the manuscript and to Drs. O. Lowry, R. Wilkinson, and B. Rosser for critical reading of the manuscript.

Correspondence should be addressed to Patti M. Nemeth, Ph.D., Department of Neurology, Box 8111 , Washington University School of Medicine, 660 S. Euclid Ave., St. Louis, MO 63110.

Copyright (C) 1989 Society for Neuroscience $0270-6474 / 89 / 072336-08 \$ 02.00 / 0$
}

During development of future fast and slow muscles in the rat, myosin undergoes a sequence of transitions that involve the embryonic, neonatal, and adult slow and fast myosin heavy chain genes (Whalen et al., 1981; Lyons et al., 1983; Periasamy et al., 1984; Mahdavi et al., 1986; Narusawa et al., 1987). These transitions presumably correlate with the emergence of functional specialization. It is, however, unclear whether the changes in cellular energy metabolism accompany the changes in myosin isoform expression. This seemed to us worth exploring because enzymatic transitions precede those of myosin isozymes in chronically stimulated adult fast muscle (Pette et al., 1983), leading to the hypothesis that changes in the "internal environment" of the muscle cell must reach a critical threshold before the genes controlling synthesis of the structural proteins are affected (Pette and Vrbová, 1985). Thus, the present study examines the relationship of metabolic enzyme profiles to myosin changes and to other changes in the cellular environment.

Although there have been a number of histochemical enzyme studies of developing muscle (Bass et al., 1970; Brooke et al., 1971; Pette, 1971; Hudlická et al., 1973; Tomanek, 1975; Curless and Nelson, 1976; Halta et al., 1978), a more precise assessment of metabolic differentiation can be achieved by enzyme quantitation. Individual fibers of the rat fast-twitch extensor digitorum longus (EDL) and slow-twitch soleus muscles were analyzed at successive stages of development using extremely sensitive and accurate microanalytic methods (Lowry and Passonneau, 1972). Adenylokinase (AK) and lactate dehydrogenase (LDH) enzymes were selected as likely correlates of contractile function because high levels of both are found in adult motor units having the fastest contraction times (Hamm et al., 1987). Furthermore, both enzymes have wide ranges in adult rat muscle, with sufficient discontinuity between fast and slow fiber groups to be useful for enzymatic fiber typing (Lowry et al., 1978; Hintz et al., 1980; Nemeth et al., 1986). Oxidative capacity was assessed by levels of malate dehydrogenase (MDH) for the citric acid cycle and of $\beta$-hydroxyacyl CoA dehydrogenase $(\beta O A C)$ for oxidation of fatty acids.

In order to relate influences of the cellular environment to the metabolic energy systems, dietary patterns and changes in thyroid hormones were considered. Dictary intake is naturally altered as the developing rat moves from a predominantly glycolytic metabolism in utero to a high-fat, low-carbohydrate diet during suckling, and finally to a diet rich in carbohydrate after weaning. The influence of emerging thyroid function on the developing fibers in suckling and adolescent rats was examined 
under euthyroid and experimental hyper- and hypothyroid conditions.

\section{Materials and Methods}

Sprague-Dawley rats (Hilltop Laboratories, Philadelphia) were killed at sequential stages of postnatal development. A single litter was used for the 0-21 d euthyroid study; other litters were added for subsequent time points. A separate litter was made hyperthyroid by injecting each pup with thyroxine $(1 \mathrm{mg} / \mathrm{kg})$ on alternate days after birth. Another litter was made hypothyroid by feeding the mother $0.05 \%$ propylthiouracil in drinking water beginning at day 12 of gestation (Gambke et al., 1983).

Animals were killed quickly (within $60 \mathrm{sec}$ ) in a $\mathrm{CO}_{2}$ euthanasia chamber before surgery. EDL and soleus muscles were isolated from the surrounding muscles in situ with the tendons intact; then whole hindlimbs containing the isolated muscles were frozen in liquid nitrogen. The frozen muscles were removed from the limbs and lyophilized for $7 \mathrm{~d}$ at $-38^{\circ} \mathrm{C}$ at a pressure less than $10^{-2}$ torr. Tissue was stored thereafter at $-80^{\circ} \mathrm{C}$ under vacuum until further processing.

Enzyme activities were measured in ng samples of individual muscle fibers by microfluorometry of the pyridine nucleotide cofactors of specific enzyme reactions. This measures changes in the oxidation or reduction of the nucleotide by capturing the remitted fluorescence of absorbed light at specific wavelengths with far greater sensitivity than does spectrophotometric absorption $(0.1-10 \mu \mathrm{M}$ with fluorometry vs 10-200 $\mu \mathrm{M}$ with spectrophotometry). When needed for low enzyme activities, a further gain in sensitivity was achieved by enzymatic cycling in which a pyridine nucleotide acts as a catalytic intermediate for 2 simultaneous oxidation and reduction reactions, generating increasingly high concentrations of reaction products. The analytical procedures have been exquisitely developed for precision, accuracy, and optimal activation by Lowry and his associates. Methods for AK, $\mathrm{LDH}$, and $\beta \mathrm{OAC}$ are from Lowry et al. (1978); those for MDH are from Hintz et al. (1980). A representative sample of fibers from each muscle was assayed. The sample size was based on previous studies showing that $10-20$ fibers of a given muscle from individual animals of the same species completely overlap in enzyme activities (e.g., Nemeth and Pette, 1981; Nemeth et al., 1986). When sufficient fiber size permitted, different enzymes were measured on the same fiber. Enzyme activities are expressed as $\mathrm{mol} / \mathrm{kg}$ dry weight $/ \mathrm{hr}$ at $20^{\circ} \mathrm{C}$.

Metabolic studies dictate the use of the histochemical or biochemical fiber type nomenclature: slow-twitch oxidative (SO), fast-twitch oxidative glycolytic (FOG), and fast-twitch glycolytic (FG). Reference is also made in this report to the myosin ATPase types I, IIa, and IIb. It is recognized, however, that an incomplete homology exists between the metabolic classification system and myosin ATPase types (Nemeth and Pette, 1981) and that a correlation between metabolic enzymes and specific myosin heavy chains (MHC) I, IIa, and IIb has not been established.

\section{Results}

Adult rat soleus muscle is known to consist mainly (85-90\%) of SO fibers, while the EDL muscle contains a mixture of fiber types, predominantly fast-twitch: approximately $59 \%$ FOG, $38 \%$ FG, and 3\% SO (Armstrong and Phelps, 1984). The 2 muscles show distinctive courses of metabolic maturation, as described below.

\section{Lactate dehydrogenase}

This enzyme is used as a marker for glycogenolytic metabolism. Figure 1 shows its developmental transitions in the fiber populations of the 2 hindlimb muscles. At birth, LDH activities are higher in EDL than in soleus muscle (on average, 38 vs $29 \mathrm{~mol} /$ $\mathrm{kg}$ dry weight $/ \mathrm{hr}$ ). Despite the fact that there is an overlap of LDH activities in the 2 fiber populations at birth, the difference between the 2 populations is highly significant $(p<.0005)$. Even at this early stage there is variability in LDH among the individual fibers of each muscle, 2.4-fold in EDL and 2.2-fold in soleus between the extreme activity levels.

The separation between the 2 populations widens gradually

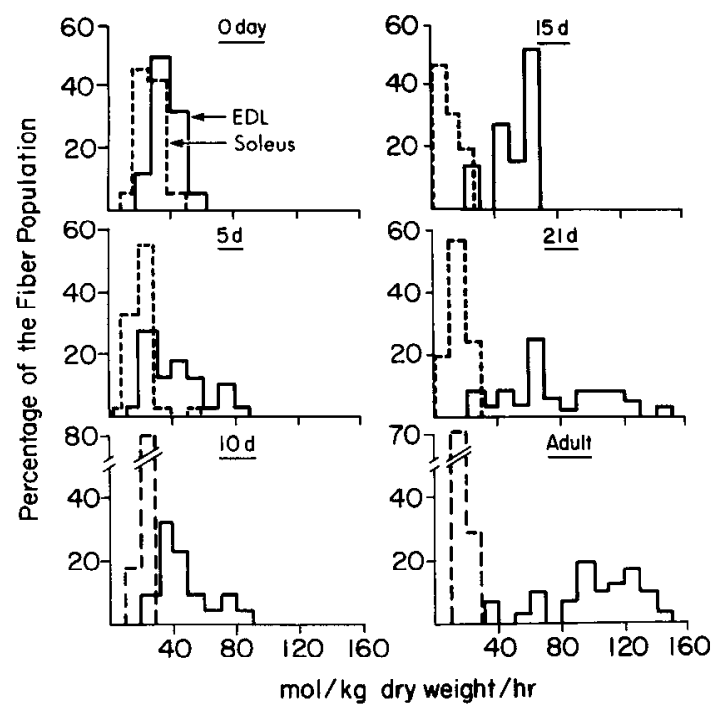

Figure 1. LDH activity levels in individual fibers of the EDL (solid lines) and soleus (dashed lines) muscles of euthyroid rat siblings at successive stages of postnatal development. The adult rat was from a separate litter. The average number of fibers analyzed from each muscle was 22 .

during the first 2 weeks, partially because of a substantial decrease in soleus levels. Then, beginning at $15 \mathrm{~d}$, a major shift upward occurs in the EDL but not in the soleus. As a result, the average value for EDL fibers is 5 -fold higher than the average for soleus fiber. This division yields the distinctive fiber profiles characteristic of the adult fiber populations of the 2 muscles.

Average LDH activity in the soleus, unlike that in the EDL, gradually decreases from birth to day 15 , at which time levels have decreased by $52 \%$. Thereafter, levels increase but remain lower than those at birth, with similar or slightly lower interfiber variation.

\section{Adenylokinase}

This is an enzyme of high-energy phosphate metabolism which covaries with $\mathrm{LDH}$, presumably because of its role in the positive modulation of enzymes of glycolysis through the production of $5^{\prime}$-AMP. As with LDH, the AK activities (Fig. 2) overlap in fibers of soleus and EDL muscles at birth, and the 2 fiber populations are significantly different $(p<0.0005)$. Again, there is a wide spectrum of activities in both muscles, particularly in the soleus where the range is nearly 6-fold. Some degree of overlap persists in the 2 fiber groups even in the adult.

The EDL fibers change only slightly in average AK activity over the first $10 \mathrm{~d}$. Then, between 10 and $21 \mathrm{~d}$, there is a major (3-fold) increase in average activity, an event which parallels that in $\mathrm{LDH}$. To establish the adult profile, average values fall and the range of activities increases.

AK values in soleus fibers have distinctly different average activities from those of EDL and change little in the first 2 weeks. A substantial rise which is proportional to that in EDL occurs at $21 \mathrm{~d}$. Thereafter, AK activity in soleus returns to birth levels.

There is a particularly wide range of AK activity in soleus fibers throughout early development, terminating in a more uniform population in the adult. Therefore, as a general rule, interfiber variability of both AK and LDH increases in the EDL to accommodate 2 major fiber types, whereas the variability of both enzymes decreases in the soleus to form a single major 


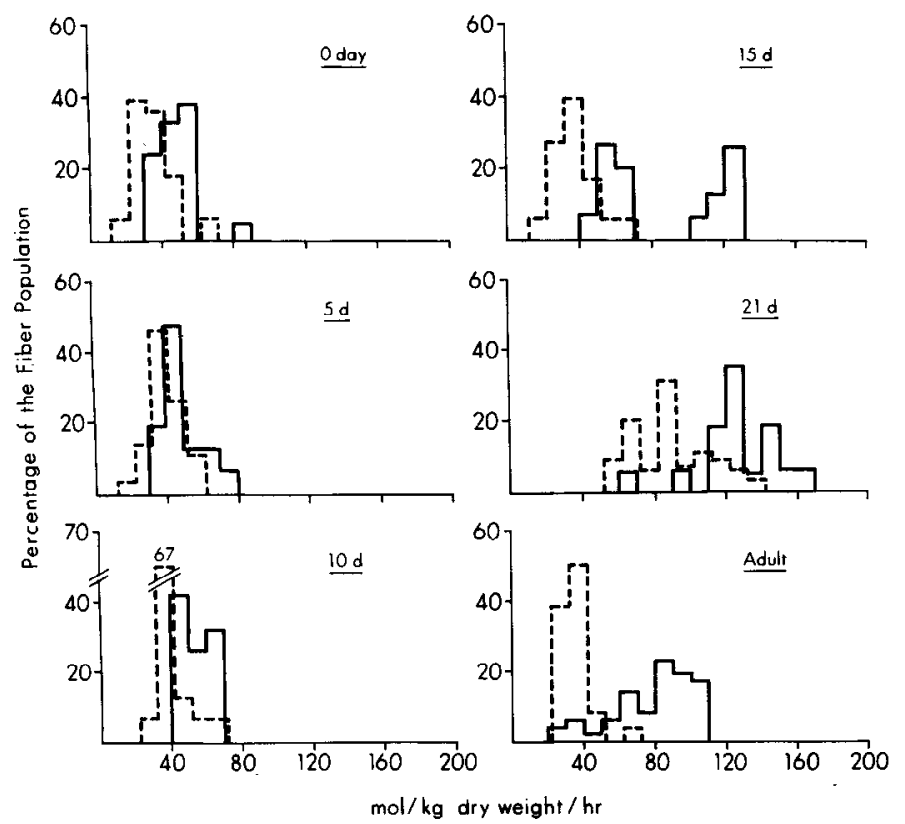

Figure 2. AK activities as in Figure 1.

type. Some of the remaining variability in the adult soleus can be attributed to the low percentage of typc FOG fibcrs in the predominantly type SO population. The same is true in the EDL with the rare type SO fibers. Examples of these rare fibers are seen in the "adult" panels in Figures 1 and 2.

\section{Malate dehydrogenase}

Oxidative capacity as judged by MDH (Fig. 3) is generally higher at birth in soleus muscle fibers than in EDL fibers. However, by $5 \mathrm{~d}$, EDL values almost completely overlap those of the
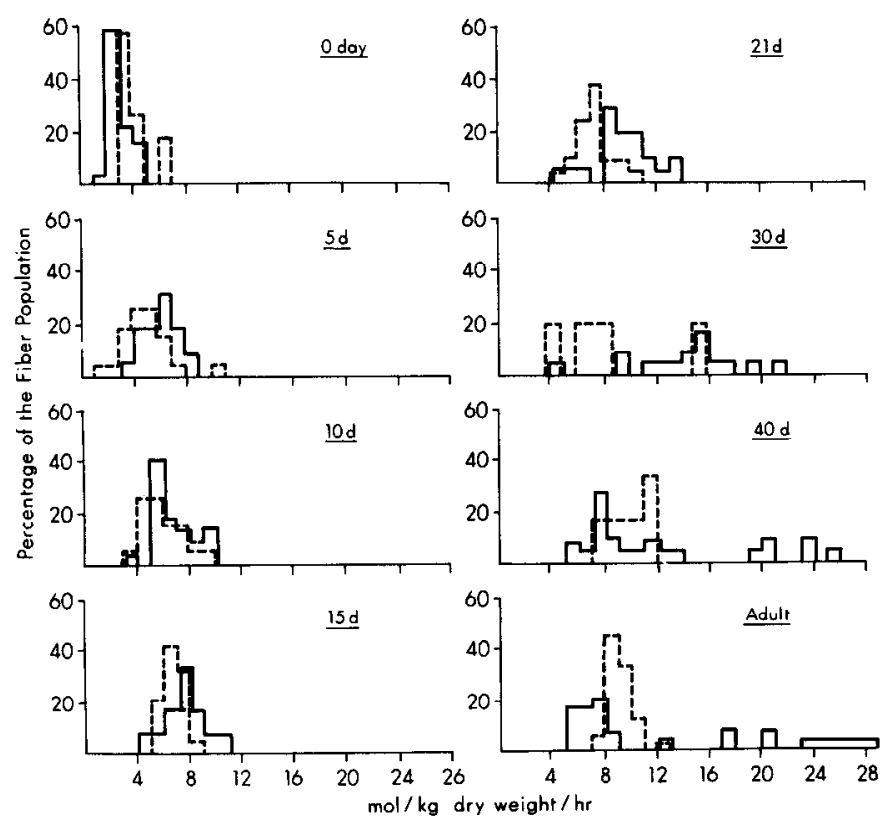

Figure 3. MDH activities as in Figure 1 except that 30 and $40 \mathrm{~d}$ rats were from a separate litter.

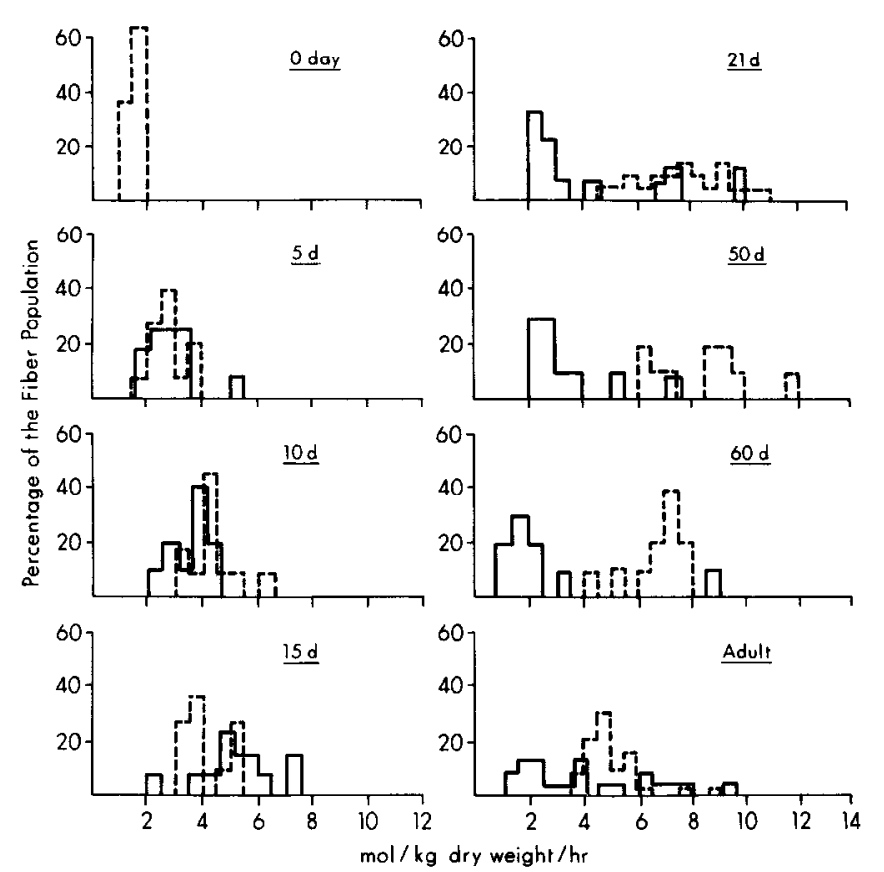

Figure 4. $\beta \mathrm{OAC}$ activities as in Figure 1 except that 50 and $60 \mathrm{~d}$ rats were from a separate litter.

soleus. Values in both muscles rise together gradually and continuously until $21 \mathrm{~d}$. Only a slight increase occurs in soleus fibers after $21 \mathrm{~d}$ to reach adult levels, as illustrated by measurements in 30- and 40-d old animals. The variability of $\mathrm{MDH}$ in the soleus is reduced considerably from birth to adulthood (see Fig. 5) to produce the single type SO fiber population.

The fiber population of the EDL, on the other hand, undergoes significant remodeling after $21 \mathrm{~d}$. While average values are higher in EDL than in soleus at all ages after birth (Fig. 3), one portion of the fibers increases in MDH activity whereas another portion decreases in activity. This produces a bimodal distribution of fibers that is clearly apparent at $40 \mathrm{~d}$ and an extremely high interfiber variation - as high as 5.3-fold in the adult. EDL fibers are divided into FG and FOG populations on the basis of low and high $\mathrm{MDH}$ activities, respectively (see below).

\section{$\beta$-hydroxyacyl-CoA dehydrogenase}

The rise in oxidative metabolism reflected by $\mathrm{MDH}$ activities in both soleus and EDL muscles is consistent with an adaptation to aerobic extrauterine environment and oxidative metabolism. Since fatty acids derived from milk and endogenous stores are the predominant source of energy in the suckling neonate, $\beta \mathrm{OAC}$ was measurcd as an enzyme marker for the oxidation of fatty acids of variable chain lengths (Fig. 4).

As with $\mathrm{MDH}, \beta \mathrm{OAC}$ activity is nearly indistinguishable in the soleus and EDL at $5 \mathrm{~d}$ (not measured in EDL at birth) and tends to increase synchronously in the 2 muscles for up to 15 d. At $21 \mathrm{~d}$ an unusually wide spectrum of activities appears in both muscles, with those in soleus fibers highest on average. The high activities are maintained in the soleus after $21 \mathrm{~d}$; in fact, they continue to rise up to $50 \mathrm{~d}$ (Fig. 4) before declining to adult levels. Activities in the EDL fibers, on the other hand, fall to nearly adult levels by $21 \mathrm{~d}$. The adult enzyme profile appears to be attained by further widening of the activities in the EDL fiber population and a decrease and narrowing of values 


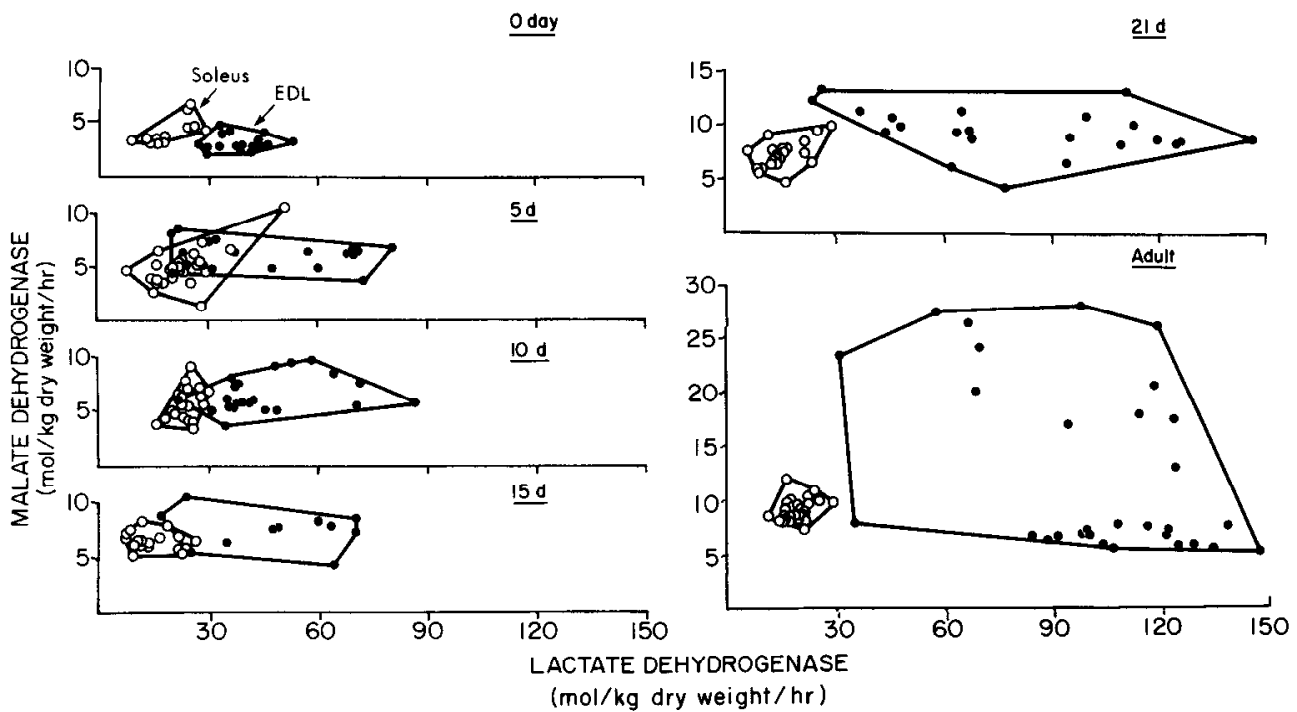

Figure 5. Profiles of the activities of oxidative and glycolytic enzymes determined on the same individual fibers of EDL (O) and soleus (O) muscles of euthyroid rat siblings at successive stages of development. Each circle represents a separate fiber. Activities are expressed as $\mathrm{mol} / \mathrm{kg}$ dry weight $/ \mathrm{hr}$. In the adult profile, SO fibers are low in LDH and intermediate in $\mathrm{MDH}$; FOG fibers are high in both $\mathrm{LDH}$ and $\mathrm{MDH}$; and FG fibers are low in $\mathrm{MDH}$ and high in LDH. in the soleus muscle to form the 3 major fiber groups: low levels in FG fibers, intermediate levels in SO, and high levels in FOG.

\section{Fiber type-specific changes}

It is often difficult to delineate fiber types on the basis of a single enzyme because of the overlapping spectrum of activities. As mentioned above, it is possible to measure different enzymes on the same individual fiber with the present techniques. The combination of $\mathrm{MDH}$ and $\mathrm{LDH}$ has proven to be useful for enzymatic fiber typing (Lowry et al., 1978; Hintz et al., 1980; Nemeth et al., 1986). Designations in adult rat muscle are: (1) Fibers very low in $\mathrm{LDH}$ and intermediate in $\mathrm{MDH}$ are type SO; (2) fibers high in both LDH and MDH are FOG; and (3) fibers high in LDH but very low in MDH are FG. The systematic development of metabolic fiber types using these criteria is shown in Figure 5. Fiber populations of the soleus and EDL are distinct at birth. Thereafter, the soleus does not undergo significant changes, only the modest reduction in the early variability in LDH and MDH among fibers and the increase in oxidative potential. The metabolic profile in general is not altered from birth nor does the profile approximate any type other than type SO.

EDL, however, does show major transitions. At birth, both LDH and MDH are low. Some fibers retain that profile during the early weeks of development, while others shift toward a profile of high LDH and low MDH by $21 \mathrm{~d}$. The latter profile defines the FG fiber type. It is at some time after $21 \mathrm{~d}$ that the highly oxidative type FOG fibers emerge. Interestingly, groups of fibcrs with high oxidative activitics (presumably FOG fibcrs) and with low oxidative activities (presumably FG fibers) are revealed by $\beta \mathrm{OAC}$ at $21 \mathrm{~d}$ (Fig. 4).

Figure 6 shows the successive changes in average values of each enzyme and serves to distinguish the major differences in the maturation of prospective fast and slow muscles. In general, the high-energy phosphate marker, AK, and glycolytic marker, $\mathrm{LDH}$, are relatively stable for the first $10 \mathrm{~d}$ and then rise abruptly. (The important exception is the decrease in LDH in the soleus.) The rise occurs at a time that coincides with a number of physiological adaptive changes (see Discussion). Thereafter, the 2 fiber populations diverge. The oxidative enzymes, $\beta \mathrm{OAC}$ and $\mathrm{MDH}$, are similar in the 2 muscle types at early times and increase together. However, it is evident here that there are fiber type-specific changes in effect for both $\mathrm{MDH}$ and $\beta \mathrm{OAC}$ after 21 or $30 \mathrm{~d}$. Fast muscle shows a sharper increase in MDH than does slow muscle. On the other hand, fast muscle has lower $\beta \mathrm{OAC}$ after $21 \mathrm{~d}$ and decreases to adult levels much sooner than does slow muscle.

\section{Influence of thyroid hormones}

It is known that thyroid hormone regulates the expression of contractile proteins in developing muscle (Gambke et al., 1983; Butler-Browne et al., 1984). To examine whether metabolic proteins match those of myosin in response to hormone changes, we compared $\mathrm{LDH}, \mathrm{AK}, \mathrm{MDH}$, and $\beta \mathrm{OAC}$ levels in the EDL muscles of euthyroid, hyperthyroid, and hypothyroid rats. Muscle fibers of hyperthyroid rats tend to reach adult enzyme levels very rapidly, while fibers of hypothyroid rats generally do not reach adult levels. The changes in $\mathrm{LDH}$, illustrated in Figure 7, are representative of this pattern. The fibers of hyperthyroid rats reach adult levels by $10 \mathrm{~d}$, and these levels subsequently do not vary. In contrast, the hypothyroid LDH values remain similar to the $10 \mathrm{~d}$ euthyroid values even at $30 \mathrm{~d}$. These results strongly suggest that the development of thyroid function is necessary for the rise in LDH to adult levels. Induced hyperthyroidism increases the rate of this process, while induced hypothyroidism inhibits it.

A situation similar to that in LDH is evident in AK and MDH enzymes (Fig. 8). The hyperthyroid state produces adult enzyme levels by $10 \mathrm{~d}$, while hypothyroidism holds levels to approximately half those of the adult. Euthyroid animals excecd hypothyroid in enzyme levels but do so at different times.

$\beta \mathrm{OAC}$ is unique in that both hyper- and hypothyroid conditions induce considerable elevation in enzyme levels, even exceeding normal adult levels (Fig. 8). This occurs as early as $10 \mathrm{~d}$. Our interpretation of this change is that in an attempt to maintain homeostasis, hypothyroid animals make use of this alternate, lipid, metabolic pathway. At 15 and 21 d, hyper- and hypothyroid rats have $\beta O A C$ levels similar to those of normal rats of that age and adults. However, $\beta O A C$ at $30 \mathrm{~d}$ is lower in hyperthyroid than in normal animals, indicating that the increased hormone level can hasten the $\beta \mathrm{OAC}$ enzyme to its lower adult level. 
Figure 6. Average enzyme activities of individual fibers in EDL $(0)$ and soleus $(O)$ muscles of euthyroid rats at successive stages of development.
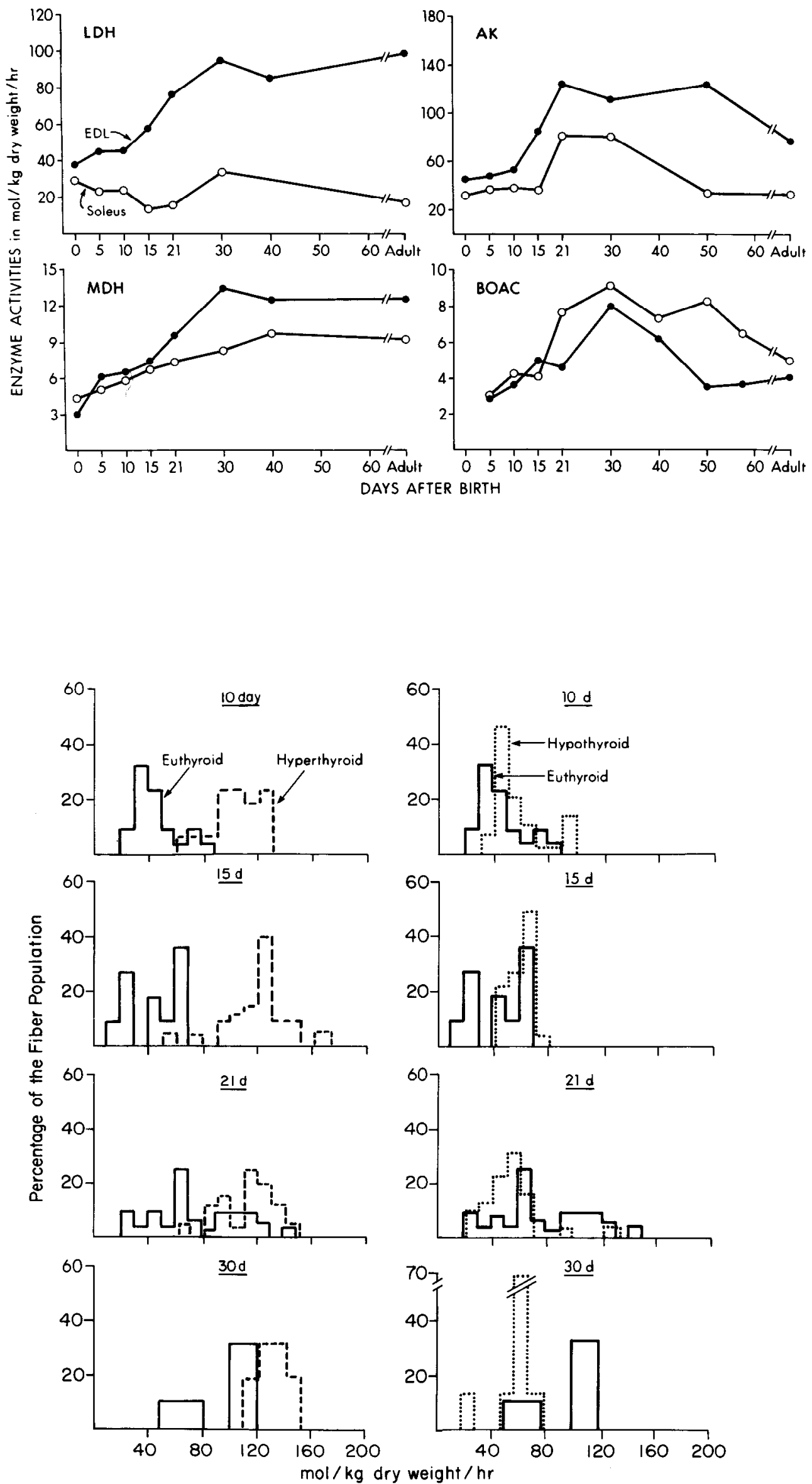

Figure 7. LDH activity levels of individual fibers in EDL muscles of euthyroid (solid lines), hyperthyroid (dashed lines), and hypothyroid (dotted lines) rat siblings. The average number of fibers analyzed from each muscle was 18 . 


\section{Discussion}

The present study outlines the changes in metabolic properties within fibers of developing fast and slow muscles in the rat. The cell-by-cell enzymatic characterization afforded by the quantitative techniques, moreover, provides a precise picture of the emergence of metabolic diversity and new information on the chronology of cellular specialization in mammalian muscle.

Our results show that at birth enzyme levels in the oxidative and glycolytic pathways as well as AK are low in all fibers of the soleus and EDL muscles. Thus, although adult skeletal muscle is the most energy-demanding tissue in the body, neonatal muscle appears to have only a modest capacity to gencratc ATP. This probably means that neonatal limb muscles can sustain contractions for only short periods of time and correlates with the finding that both the soleus and EDL are activated phasically and at low rates in the baby rat (Navarrete and Vrbová, 1983; Vrbová et al., 1985). Neonatal muscles also have prolonged velocities of shortening and relaxation (Close, 1972), suggesting that the contractile machinery of the newborn rat is adapted to make economical use of restricted supplies of energy so that fatigue does not inhibit essential muscle performance. Since slow myosin has a low energy cost for force production (Crow and Kushmerick, 1982), the early expression of slow MHC in fetal and neonatal muscle (Narusawa et al., 1987) is consistent with this interpretation. So is the finding by Whalen (1980) that the actin-activated ATPase activity of cultured rat muscle, which predominantly expresses embryonic myosin, is low and of the order of adult slow muscle.

We show that small differences in levels of $\mathrm{LDH}$ and AK activity in the neonatal soleus and EDL herald the emergence of metabolic diversity between fast and slow muscles. Moreover, individual fiber analyses demonstrate that there is significant variability of $\mathrm{AK}, \mathrm{LDH}, \mathrm{MDH}$, and $\beta \mathrm{OAC}$ activities among fibers of the EDL and soleus at this time. Interfiber variations for all 4 enzymes in the neonatal soleus exceed those of the adult, suggesting that this adult muscle, which is composed of a relatively pure fiber type, develops from a tissue with a more diverse potential.

Specialization of fast fibers into oxidative and glycolytic phenotypes occurs at a subsequent phase of development. This begins to occur between 10 and $21 \mathrm{~d}$ when mean LDH, AK, and $\beta \mathrm{OAC}$ values in the EDL abruptly increase (Figs. 1, 2, 4, 6). Accompanying this, there is an expansion in the range of activities of these enzymes such that populations of fibers with high and low $\mathrm{LDH}, \mathrm{AK}$, and $\beta \mathrm{OAC}$ values can be recognized. Therefore, we conclude that oxidative versus glycolytic specialization of fast fibers occurs at this time as MHCs and patterns of energy metabolism adjust to specific energy requirements.

A remarkable feature of the development of metabolic diversity is that in both the soleus and the EDL the patterns of activity of $\mathrm{LDH}, \mathrm{AK}, \mathrm{MDH}$, and $\beta \mathrm{OAC}$ follow independent pathways to maturity, implying that distinct regulatory mechanisms govern the development of each enzyme system in the 2 muscles (Fig. 6).

Whether the nerve tailors the initial differences in $\mathrm{LDH}$ and AK activity between the neonatal soleus and EDL is uncertain. It now appears unlikely that the nerve is responsible for determining initial differences of MHC expression in primordial muscle cells of the chick embryo. Early patterns of diversity precede innervation (Butler et al., 1982; Phillips and Bennett, 1984) and are faithfully reproduced in muscle cultures (Miller et al., 1985;
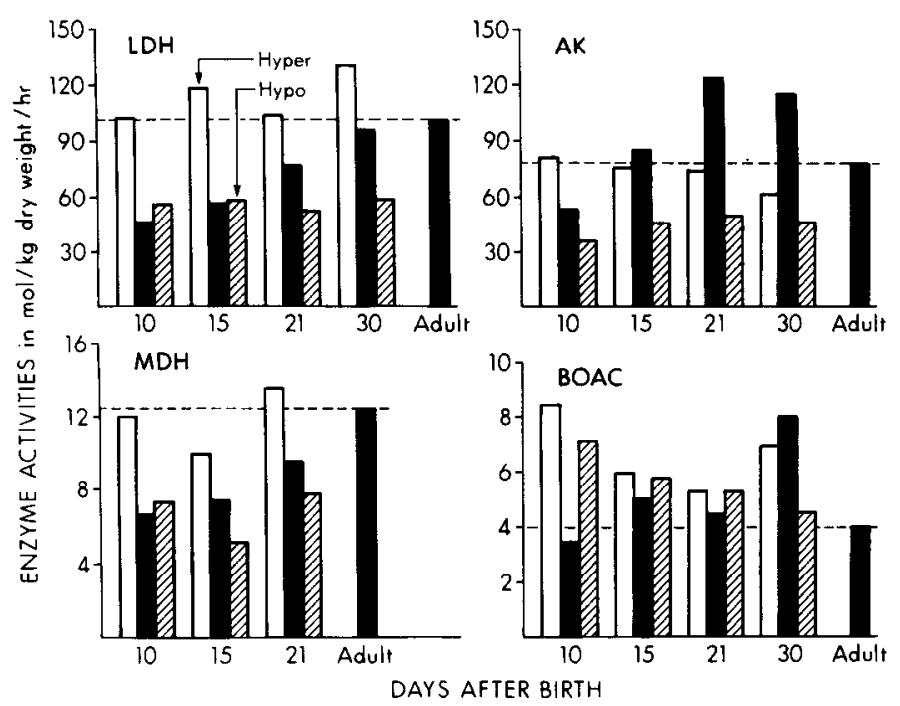

Figure 8. Average enzyme activities of individual fibers in EDL muscle of hyperthyroid (white bars) and hypothyroid (hatched bars) rat siblings compared to euthyroid (black bars) rats of the same postnatal age. Dashed lines indicate the relationship to adult EDL values.

Crow and Stockdale, 1986). Moreover, a number of studies (Rubinstein and Kelly, 1981; Butler et al., 1982; Laing and Lamb, 1983; Phillips and Bennett, 1984) suggest that as the nerve invades the muscle primordium it must recognize distinctions in the membrane properties of different classes of myotubes and integrates itself with a predetermined program of muscle specialization. The commitment to specific patterns of muscle differentiation therefore appears to be intrinsically determined very early in myogenesis. It seems probable to us that this decision must have global molecular consequences for the developing muscle cell and must involve not only programs of MHC expression but parallel changes in membrane properties as well as key metabolic pathways. Thus, the metabolic differences between the neonatal soleus and EDL may be as much the product of intrinsic determination as of neural control. Our own studies are consistent with this interpretation, for we have found that activities of $\mathrm{LDH}, \mathrm{MDH}$, phosphorylase, and AK differ considerably between single myotubes dissected from aneural primary cultures of rat skeletal muscle (Nemeth et al., 1985).

After birth, neural and thyroidal regulation appear to be progressively imposed on the evolving patterns of metabolic diversity in the soleus and EDL. This can be recognized in the development of $\mathrm{LDH}$ activity. In the EDL, the $\mathrm{M}$ (muscle) subunit of $\mathrm{LDH}$ is exclusively expressed throughout development (Margreth, 1970). Levels of LDH activity slowly increase between birth and $10 \mathrm{~d}$ and then surge from 10 to $30 \mathrm{~d}$ (Fig. 1). This surge coincides with the devclopment of thyroid function (Gambke et al., 1983; Chizzonite and Zak, 1984) and also with the development of type IIb MHC expression in the EDL (Russell et al., 1988; Rubinstein et al., 1988). The surge of LDH activity does not occur in hypothyroid animals but occurs precociously in hyperthyroid animals (Fig. 8). Expression of IIb $\mathrm{MHC}$ is also uniquely sensitive to thyroid regulation during the same period (Gambke et al., 1983; Butler-Browne et al., 1984; Russell et al., 1988; Rubinstein et al., 1988) suggesting that actions of the thyroid are designed to optimally coordinate the contractile and metabolic development of these enzyme systems 
in the EDL. These developmental events do not appear to be closely tied to neural activity since accumulation of LDH (M) continues to rise in the denervated EDL (Margreth, 1970), much as the accumulation of IIb MHC progresses after denervation (Rubinstein et al., 1988).

By contrast, a qualitative rather than quantitative change of LDH activity occurs in the developing soleus as the $H$ (heart) subunit characteristic of the adult soleus progressively replaces the M subunit that is expressed initially (Margreth, 1970). The beginnings of this switch can be detected by $5 \mathrm{~d}$. Significantly, the transition stops abruptly if the developing soleus is denervated (Margreth, 1970), revealing that $\mathrm{LDH}(\mathrm{H})$ activity in this slow-twitch muscle is closely tied to neural control. During the same period, accumulation of slow/B MHC in the soleus is also sensitive to neural control (Narusawa et al., 1987), implying that in this muscle the nerve rather than thyroid hormone plays a central role in coordinating contractile and metabolic differentiation.

One possible interpretation of these results is that in the differentiating soleus the development of tonic, postural activity by the nerve (Navarrete and Vrbová, 1983; Vrbová et al., 1985) protects and amplifies an intrinsic program of slow phenotypic development and overrides influences of the thyroid on this muscle. Conversely, the EDL may be intrinsically more sensitive to thyroid control, favoring fast phenotypic development and overriding neurally imposed influences (see Rubinstein et al., 1988).

The developmental pattern of $\beta \mathrm{OAC}$ activity indicates that in addition to thyroidal and neural controls another factor, substrate availability, plays a significant role in determining prevailing levels of $\beta$-oxidative enzyme activity in developing limb muscle. The neonate arrives suddenly into an aerobic environment where oxidation of fatty acids and ketone bodies derived from milk (rat milk is $12.5 \%$ lipid; Dymsza et al., 1964) and from fat stores becomes the major source of energy metabolism (Greengard, 1971; Kimura and Warshaw, 1983). Consequently, total blood ketone concentrations are 6 times higher and free fatty acids are 3 times higher during suckling than in the adult. These values drop after weaning at approximately $25 \mathrm{~d}$ (Page et al., 1971). During the same period, fibers of the soleus and EDL progressively increase oxidative enzyme levels, particularly $\beta \mathrm{OAC}$ levels (Fig. 4), indicating that they are adapted to make use of the available lipid substrate for energy metabolism. In view of the high yield of ATP per molecule of starting substrate, this has obvious advantages for the young animal.

$\beta O A C$ activity slowly declines in the soleus and EDL after weaning. We speculate that this occurs as stores of "puppy fat" are burned off. Consistent with this interpretation, we find that if rats are maintained on a high-fat dietary supplement after weaning, high lcvels of $\beta \mathrm{OAC}$ activity arc maintained in both muscles. By contrast, levels of other enzymes are not altered by this change of diet (P. Nemeth, B. Rosser, and A. Kelly, unpublished observations).

As we have noted, in addition to $\mathrm{LDH}$ activity, $\mathrm{AK}$ activity distinguishes the soleus and EDL at birth. Levels of AK are more than doubled in both the soleus and EDL between 10 and 21 d. However, this is only a transient phenomenon in the soleus, as levels decline to adult values by $50 \mathrm{~d}$. The rise may be attributable in part to the coincident development of thyroid function, since we show that $\mathrm{AK}$ activity is sensitive to thyroid control (Fig. 8). So far as we know, influences of neurally imposed activity on AK levels in developing muscle have yet to be explored. High levels of AK activity are correlated with fast twitch times in mature muscle (Hamm et al., 1987). In the maturing soleus, twitch times are temporarily faster between 15 and $40 \mathrm{~d}$ and then decline to mature rates (Close, 1972). This implies that contractile function and AK levels are closely related in this developing muscle. Significantly, levels of AK rise in the entire population of fibers in the soleus during the critical 15 to $40 \mathrm{~d}$ period, suggesting that the faster twitch characteristics of the adolescent soleus may involve the $60 \%$ population of slow fibers as well as the $40 \%$ population of fast IIa fibers that comprise the muscle at this time (Kugelberg, 1976). Metabolic characteristics of type I fibers in the adolescent soleus are not equivalent to those of the adult, and their twitch properties likewise may not be equivalent.

In summary, we find some degree of metabolic specialization manifest by the time of birth. This may be programmed by means either intrinsic or extrinsic to the muscle cell. Further amplification of these metabolic differences in maturing muscle then appears to be regulated by multiple factors including the imposition of varied patterns of neural stimulation, differential use of available metabolic substrate, and response to hormonal stimulation.

\section{References}

Armstrong, R. B., and R. O. Phelps (1984) Muscle fiber type composition of the rat hindlimb. Am. J. Anat. 171: 259-272.

Bass, A., G. Lusch, and D. Pette (1970) Postnatal differentiation of the enzyme activity pattern of energy supplying metabolism in slow (red) and fast (white) muscles of chicken. Eur. J. Biochem. 13: 289292.

Brooke, M. H., G. Williamson, and K. K. Kaiser (1971) The behavior of four fiber types in developing and re-innervated muscle. Arch. Neurol. Chicago 25: 360-366.

Butler, J., E. D. Cosmos, and J. Brierly (1982) Differentiation of muscle fiber types in aneurogenic brachial muscles of the chick embryo. J. Exp. Zool. 224: 64-80.

Butler-Browne, G. S., D. Herlicoviez, and R. G. Whalen (1984) Effects of hypothyroidism on myosin isozyme transitions in developing rat muscle. FEBS Lett. 166: 71-75.

Chizzonite, R. A., and R. Zak (1984) Regulation of myosin isoenzyme composition in fetal and neonatal rat ventricle by endogenous thyroid hormones. J. Biol. Chem. 259: 12628-12632.

Close, R. (1972) Dynamic properties of mammalian skeletal muscles. Physiol. Rev. 52; 129-197.

Crow, M. T., and M. J. Kushmerick (1982) Chemical energetics of slow and fast twitch muscles of the mouse. J. Gen. Physiol. 79: 149166.

Crow, M. T., and F. E. Stockdale (1986) Myosin expression and specialization among earliest muscle fibers of the developing avian limb. Dev. Biol. 113: 238-254.

Curless, R. A., and M. B. Nelson (1976) Developmental patterns of rat muscle histochemistry. J. Embryol. Exp. Morphol. 36: 335-363.

Dymsza, H. A., D. M. Czajka, and S. A. Miller (1964) Influence of artificial diet on weight gain and body composition of the neonatal rat. J. Nutr. $84: 100-106$.

Gambke, B., G. Lyons, J. Haselgrove, A. Kelly, and N. Rubinstein (1983) Thyroid and neural control of myosin transitions. FEBS Lett. 156: 335-339.

Greengard, O. (1971) Enzymatic differentiation in mammalian tissues. Essays Biochem. 7: 159-205.

Halta, M., O. Berlin, II. Schucht, and P. Sourander (1978) Postnatal differentiation and growth of skeletal muscle fibers in normal and undernourished rats. J. Neurol. Sci. 36: 25-39.

Hamm, T. M., P. M. Nemeth, L. Solanki, D. A. Gordon, R. M. Reinking, and D. G. Stuart (1987) Association between biochemical and physiological properties in single motor units. Muscle and Nerve 11:95104.

Hintz, C. S., C. V. Lowry, K. K. Kaiser, D. McKee, and O. H. Lowry (1980) Enzyme levels in individual rat muscle fibers. Am. J. Physiol. 239 (Cell Physiol. 8): C58-C65. 
Hudlická, O., D. Pette, and H. Staudte (1973) The relation between blood flow and enzymatic activities of slow and fast muscles during development. Pfluegers Arch. 343: 341-356.

Kimura, R. E., and J. B. Warshaw (1983) Metabolic adaptations of the fetus and newborn. J. Pediatr. Gastroenterol. Nutr. 2 (Suppl. 1): S12-S15.

Kugelberg, E. (1976) Adaptive transformation in rat soleus motor units during growth. J. Neurol. Sci. 27: 269-289.

Laing, N. G., and A. H. Lamb (1983) The distribution of muscle fiber types in chick embryo wings transplanted to the pelvic region is normal. J. Embryol. Exp. Morphol. 78: 67-82.

Lowry, C. V., J. S. Kimmey, S. Felder, M. M.-Y. Chi, K. Kaiser, P. M. Passonneau, K. A. Kirk, and O. H. I owry (1978) Enzyme patterns in single human muscle fibers. J. Biol. Chem. 253: 8269-8277.

Lowry, O. H., and J. V. Passonneau (1972) A Flexible System of Enzymatic Analysis, Academic, New York.

Lyons, G. E., J. Haselgrove, A. M. Kelly, and N. Rubinstein (1983) Myosin transitions in developing fast and slow muscles of the rat hind limb. Differentiation 25: 168-175.

Mahdavi, V., E. E. Strehler, M. Periasamy, D. Wieczorek, S. Izumo, and B. Nadal-Ginard (1986) Sarcomeric myosin heavy chain gene family: Organization and pattern of expression. Med. Sci. Sports Exerc. 18: 299-308.

Margreth, A. (1970) Developmental patterns of LDH isozymes in fast and slow muscles of the rat. Arch. Biochem. Biophys. 141:374-377.

Miller, J. B., M. T. Crow, and F. E. Stockdale (1985) Slow and fast myosin heavy chain content defines three types of myotubes in early muscle cell cultures. J. Cell Biol. 101: 1643-1650.

Narusawa, M., R. Fitzsimons, S. Izumo, B. Hadal-Ginard, N. A. Rubinstein, and A. M. Kelly (1987) Slow myosin in developing rat skeletal muscle. J. Cell Biol. 104: 447-459.

Navarrete, R., and G. Vrbová (1983) Changes of activity patterns in slow and fast muscles during postnatal development. Dev. Brain Res. 8: $11-19$.

Nemeth, P., and D. Pette (1981) Succinate dehydrogenase activity in fibres classified by myosin AT Pase in three hindlimb muscles of the rat. J. Physiol. (Lond.) 320: 73-80.

Nemeth, P. M., L. Solanki, and J. C. Lawrence, Jr. (1985) Control of enzyme activities in individual myotubes cultured without nerve. Am. J. Physiol. 249 (Cell Physiol. 18): C313-C317.

Nemeth, P. M., I. Solanki, D. A. Gordon, T. M. Hamm, R. M. Reinking, and D. G. Stuart (1986) Uniformity of metabolic enzymes within individual motor units. J. Neurosci. 6: 892-898.
Page, A. M., A. H. Krebs, and D. H. Williamson (1971) Activities of enzymes of ketone body utilization in brain and other tissues of suckling rats. Biochem. J. 121: 49-53.

Periasamy, M., D. F. Wieczorek, and B. Nadal-Ginard (1984) Characterization of a developmentally regulated perinatal myosin heavy chain gene expressed in skeletal muscle. J. Biol. Chem. 259: 1357313578.

Pette, D. (1971) Metabolic differentiation of distinct muscle types at the level of enzymatic organization. In Muscle Metabolism during Exercise, B. Pernow and B. Saltin, eds., pp. 33-47, Plenum, New York.

Pette, D., and G. Vrbová (1985) Invited review: Neural control of phenotypic expression in mammalian muscle fibers. Muscle and Nerve 8: 676-689.

Pette, D., W. Ramirez, W. Müller, R. Simon, G. U. Exner, and R. Hildebrand (1983) Influence of intermittent long term stimulation on contractile, histochemical and metabolic properties of fiber populations in fast and slow rabbit muscles. Pfluegers Arch. 361: 1-7.

Phillips, W. D., and M. R. Bennett (1984) Differentiation of fiber types in wing muscle during embryonic development: Effect of neural tube removal. Dev. Biol. 106: 457-468.

Rubinstein, N. A., and A. M. Kelly (1981) Development of muscle fiber specialization in the rat hind limb. J. Cell Biol. 90: 128-144.

Rubinstein, N. A., G. E. Lyons, and A. M. Kelly (1988) Hormonal control of myosin heavy chains during development of skeletal muscle. Ciba Foundation Symposium, Plasticity of the Neuromuscular System, Wiley, Chichester, U.K.

Russell, S. D., N. Cambon, B. Nadal-Ginard, and R.G. Whalen (1988) Thyroid hormorie induces a nerve-independent precocious expression of fast myosin heavy chain mRNA in rat hindlimb skeletal muscle. J. Biol. Chem. 263: 6370-6374.

Tomanek, R. J. (1975) A histochemical study of postnatal differentiation of skeletal muscle with reference to functional overload. Dev. Biol. 42: 305-314.

Vrbová, G., R. Navarrete, and M. Lowrie (1985) Matching of muscle properties and motoneuron firing patterns during early stages of development. J. Exp. Biol. 115: 113-123.

Whalen, R. G. (1980) Contractile protein isozymes in development: The embryonic phenotype. In Plasticity of Muscle, D. Pette, ed., pp. 177-191, de Gruyter, New York.

Whalen, R. G., S. M. Sell, G. S. Butler-Browne, K. Swart7, P. Vouveret and J. Pinset-Harstrom (1981) Three myosin heavy-chain isozymes appear sequentially in rat muscle development. Nature 292: 805-809. 